\title{
Concomitant Sleep Apnea and Diabetes Mellitus Are Independently Associated with Cardiovascular Events and Hospitalization for Heart Failure after Coronary Artery Bypass Grafting
}

\author{
Aye-Thandar Aung \\ National University of Singapore \\ Chieh-Yang Koo \\ National University of Singapore \\ Wilson W Tam \\ National University of Singapore \\ Zhengfeng Chen \\ National University of Singapore \\ William Kristanto \\ $\mathrm{Ng}$ Teng Fong General Hospital \\ Hui-Wen Sim \\ National University of Singapore \\ Pipin Kojodjojo \\ $\mathrm{Ng}$ Teng Fong General Hospital \\ Theodoros Kofidis \\ National University of Singapore \\ Chi-Hang Lee ( $\nabla$ mdclchr@nus.edu.sg ) \\ National University Heart Centre https://orcid.org/0000-0001-8777-2705
}

Original investigation

Keywords: obstructive sleep apnea, diabetes mellitus, major adverse cardiac and cerebrovascular events, coronary artery bypass grafting, heart failure

Posted Date: May 29th, 2020

DOI: https://doi.org/10.21203/rs.3.rs-30720/v1

License: (c) (i) This work is licensed under a Creative Commons Attribution 4.0 International License.

Read Full License 


\section{Abstract}

\section{Background}

Sleep apnea is a prevalent condition and an indicator of a poor outcome after coronary artery bypass grafting (CABG). Previous studies have not explored the relative and combined effects of sleep apnea with diabetes mellitus (DM) on cardiovascular outcomes in patients undergoing CABG.

\section{Methods}

In this secondary analysis of data from the SABOT study, 1007 patients were reclassified into four groups based on their sleep apnea and DM statuses, yielding 295, 218, 278, and 216 patients in the sleep apnea (+) DM (+), sleep apnea (+) DM (-), sleep apnea (-) DM (+), and sleep apnea (-) DM (-) groups, respectively. The primary end point was a major adverse cardiac and cerebrovascular event (MACCE), which encompasses cardiovascular mortality, non-fatal myocardial infarction, non-fatal stroke, and unplanned revascularization. Hospitalization for heart failure was a key secondary end point.

Results

After a mean follow-up period of 2.1 years, the crude incidence of MACCEs was $18 \%$ in the sleep apnea (+) DM (+), $11 \%$ in the sleep apnea (+) DM (-), 13\% in the sleep apnea (-) DM (+), and $5 \%$ in the sleep apnea (-) DM (-) groups. Using sleep apnea (-) DM (-) as the reference group, a Cox regression analysis indicated that sleep apnea (+) and DM (+) independently predicted MACCEs (adjusted hazard ratio, 3.2; $95 \%$ confidence interval, $1.7-6.2 ; p=0.005$ ) and hospitalization for heart failure (adjusted hazard ratio, 12.6; $95 \%$ confidence interval, 3.0-52.3; $p<0.001$ ) after adjusting for age, sex, body mass index, left ventricular ejection fraction, hypertension, chronic kidney disease, and excessive daytime sleepiness.

Conclusion

Sleep apnea and DM have independent negative effects on the prognosis of patients undergoing nonemergent CABG.

Clinical Trial Registration

ClinicalTrials.gov identification no. NCT02701504

\section{Background}

Diabetes mellitus (DM) is a well-established risk factor and prognostic indicator of cardiovascular outcomes. Evidence suggests that patients with DM and advanced coronary artery disease would benefit more from coronary artery bypass grafting $(C A B G)$ than from percutaneous coronary intervention $[1,2]$. However, data from long-term follow-up studies have revealed independent associations of DM with the occurrence of death and adverse cardiovascular events, even among patients treated with CABG [3-8]. 
Patients with other comorbid conditions, such as renal insufficiency and peripheral vascular disease, have particularly high cardiovascular risks $[3,6]$. Consequently, interest in the cardiovascular effects of concomitant comorbid conditions in patients with DM is increasing.

Sleep apnea has been identified as an important comorbid condition that is closely associated with DM $[9,10]$. The reported prevalence of sleep apnea in patients with DM ranged from 24-61\% [9]. Sleep apnea is a chronic sleep disorder characterized by recurrent upper airway collapse during sleep, which leads to intermittent hypoxemia and hypercapnia. The sympathetic activation, blood pressure surges, and hypercoagulability caused by sleep apnea have been postulated as mediators of an increased cardiovascular risk [11]. Numerous studies have demonstrated the associations of sleep apnea with various short- and long-term complications after CABG, including new-onset atrial fibrillation, respiratory complications, hospital readmission, and major adverse cardiac and cerebrovascular events (MACCEs) [12-15]. These findings were corroborated by the recently published results of the SABOT (Sleep Apnea and Bypass Operation) study, which revealed an association of sleep apnea with a 1.57-fold increase in the risk of developing MACCEs [16].

As both sleep apnea and DM are prevalent in patients undergoing CABG, it is essential to determine the prognostic effects of these concomitant disorders. In this secondary analysis of data from the SABOT study, we reported the relative and combined effects of sleep apnea and DM on the occurrence of MACCEs and hospitalization for heart failure in patients undergoing non-emergent CABG.

\section{Methods}

\section{Study Design}

The SABOT study was a prospective, observational study that aimed to evaluate the effect of sleep apnea on the cardiovascular outcomes of patients undergoing CABG. Details regarding the methodology, patient selection, and results of the SABOT study were recently published [16]. Briefly, patients aged 1890 years who were scheduled to undergo non-emergent CABG were invited to participate in the study. The exclusion criteria included known sleep apnea on continuous positive airway pressure therapy, cardiogenic shock on mechanical ventilation and/or intra-aortic balloon pump, ongoing heart failure exacerbation requiring oxygen supplementation, perceived high risk of malignant arrhythmia, long-term use of a-blocker therapy, and severe chronic pulmonary disease. Diagnoses of DM were made by a physician based on the standard criteria [17]. The study protocol was approved by the local institutional review board (Domain Specific Review Board-C, National Healthcare Group). All participants provided written informed consent. The SABOT study has been registered with ClinicalTrials.gov (NCT02701504).

All patients who consented to participate were asked to complete the Epworth Sleepiness Scale questionnaire and the Berlin questionnaire before the overnight sleep study [18, 19]. All participants underwent an in-hospital overnight sleep study using a United States Food and Drug Administrationapproved wrist-worn portable device (Watch-PAT 200, Itamar Medical, Caesarea, Israel), which has been 
validated through in-laboratory polysomnography testing [20]. The Watch-PAT 200 measures the peripheral arterial tone (PAT), a marker of changes in arterial pulsatile volume in the finger that are regulated by a-adrenergic nerve activity in the vascular smooth muscle. This parameter reflects the sympathetic nervous system activity and was shown to be highly correlated with polysomnography data in a previous meta-analysis of 14 studies [apnea-hypopnea index $(\mathrm{AHI}), \mathrm{r}=0.893,95 \%$ confidence interval: $0.857-0.920, p<0.001]$ [20]. The Watch-PAT 200 also measures three additional channels: the heart rate (derived from the PAT signal), pulse oximetry, and actigraphy (via a built-in actigraph). Subsequently, the device uses proprietary algorithms to estimate the PAT signal amplitude, increases in heart rate and desaturation, $\mathrm{AHI}$, oxygen desaturation index, and respiratory disturbance index. The participants were classified according to the presence or absence of sleep apnea, defined respectively as a Watch-PAT AHI of $\geq 15$ or $<15$ events per hour.

In this pre-specified secondary analysis, 1007 patients from the SABOT study were reclassified into four groups according to their sleep apnea and DM statuses. All participants were followed using a combination of clinic visits, telephone contacts, and medical record reviews. All the reported outcome events were collected and adjudicated by an independent committee that was blinded to the patients' characteristics and sleep study results.

\section{Outcomes}

The pre-specified primary endpoint of this study was MACCEs, defined as the four-component composite of cardiovascular mortality, non-fatal myocardial infarction, non-fatal stroke, and unplanned revascularization. The secondary endpoints included hospitalization for heart failure, all-cause mortality, and sudden cardiac death or resuscitated cardiac arrest. All endpoint events were defined according to the Standardized Data Collection for Cardiovascular Trials Initiative [21]. Clinical event data were collected by a team blinded to the sleep study results.

\section{Statistical analysis}

Categorical variables are presented as frequencies and percentages, and differences between the four groups were evaluated using the $\chi^{2}$ test. Continuous variables with normally distributed data were summarized and compared using the independent samples t-test and are presented as means with standard deviations. Continuous variables with skewed data were compared using the Mann-Whitney U test and are presented as medians with interquartile ranges. Kaplan-Meier cumulative incidence curves for the incidence of MACCEs, all-cause mortality, cardiovascular mortality, non-fatal myocardial infarction, non-fatal stroke, unplanned revascularization, and hospitalization for heart failure were constructed and compared between the four groups using the log-rank test. The time to occurrence of MACCEs was compared between the four groups using a Cox proportional hazards regression analysis after adjusting for potential confounders. The following covariates were included in the multivariable logistic models: age, sex, body mass index, hypertension, left ventricular ejection fraction, and excessive 
daytime sleepiness. Subsequently, the hazard ratios and $95 \%$ confidence intervals were calculated. A similar Cox proportional hazards model-based analysis was performed to compare the incidence of secondary endpoints between the four groups. The SPSS Statistics 25 software program (IBM Corp., Armonk, NY, USA) was used to calculate the descriptive statistics, obtain the Kaplan-Meier cumulative incidence curves, and perform the Cox regression analyses. All analyses were two-sided and $p$ values of < 0.05 were considered to be statistically significant.

\section{Results}

\section{Baseline demographic and clinical characteristics}

Between November 2013 and December 2018, 1106 patients were prospectively enrolled into the SABOT study. Of them, 1007 patients were included in this secondary analysis. First they were reclassified into four groups based on their sleep apnea (+/-) and DM (+/-) status as follows:

1. 295 patients $(29.3 \%)$ in the sleep apnea (+) DM (+) group,

2. 218 patients $(21.6 \%)$ in the sleep apnea $(+)$ DM $(-)$ group,

3. 278 patients $(27.6 \%)$ in the sleep apnea $(-)$ DM (+) group, and

4. 216 patients $(21.5 \%)$ in the sleep apnea (-) DM (-) group.

The baseline demographic and clinical characteristics of the four groups are listed in Table 1. Approximately half of the patients in each of the two DM (+) groups were receiving insulin therapy. Patients in the sleep apnea (+) DM (+) and sleep apnea (-) DM (+) groups had similar glycosylated hemoglobin levels $(7.7 \pm 1.5 \%$ versus $7.7 \pm 1.7 \%, p=0.594)$. Patients in both $D M(+)$ groups were more likely to be female, of non-Chinese ethnicity, and non-smokers relative to those in the DM (-) groups. Both DM (+) groups also had a higher prevalence of hyperlipidemia, hypertension, and chronic kidney disease, regardless of the sleep apnea status. Moreover, patients in both sleep apnea (+) groups had a higher body mass index than those in the sleep apnea $(-)$ groups, regardless of the DM status. 
Table 1

Baseline demographic and clinical characteristics

\begin{tabular}{|c|c|c|c|c|c|}
\hline \multirow[t]{3}{*}{ Characteristics } & $\begin{array}{l}\text { Sleep } \\
\text { apnea (+) }\end{array}$ & $\begin{array}{l}\text { Sleep apnea } \\
(+) \text { DM }(-)\end{array}$ & $\begin{array}{l}\text { Sleep apnea } \\
(-) \text { DM }(+)\end{array}$ & $\begin{array}{l}\text { Sleep } \\
\text { apnea (-) }\end{array}$ & $\begin{array}{l}P \\
\text { value }\end{array}$ \\
\hline & $\mathrm{DM}(+)$ & $(n=218)$ & $(n=278)$ & $\mathrm{DM}(-)$ & \\
\hline & \multicolumn{3}{|l|}{$(n=295)$} & \multicolumn{2}{|l|}{$(n=216)$} \\
\hline Age, median (IQR), years & $\begin{array}{l}\text { 62 (57- } \\
67)\end{array}$ & $62(56-68)$ & $62(56-68)$ & $\begin{array}{l}60(56- \\
66.75)\end{array}$ & 0.341 \\
\hline Male sex, n (\%) & $\begin{array}{l}245 \\
(83.1)\end{array}$ & $197(90.4)$ & $231(83.1)$ & $\begin{array}{l}198 \\
(91.7)\end{array}$ & 0.004 \\
\hline \multicolumn{6}{|l|}{ Ethnicity, n (\%) } \\
\hline Chinese & $\begin{array}{l}191 \\
(64.7)\end{array}$ & $147(67.4)$ & $158(56.8)$ & $\begin{array}{l}156 \\
(72.2)\end{array}$ & 0.017 \\
\hline Malay & $52(17.6)$ & $41(18.8)$ & $63(22.7)$ & 38 (17.6) & \\
\hline Indian & $34(11.5)$ & $16(7.3)$ & $42(15.1)$ & $15(6.9)$ & \\
\hline Others & $18(6.1)$ & $14(6.4)$ & $15(5.4)$ & $7(3.2)$ & \\
\hline \multicolumn{6}{|l|}{ Clinical measurements } \\
\hline $\begin{array}{l}\text { Systolic blood pressure, } \\
\text { mean (SD), mm Hg }\end{array}$ & $126(20)$ & $126(18)$ & $127(19)$ & $125(19)$ & 0.803 \\
\hline $\begin{array}{l}\text { Diastolic blood pressure, } \\
\text { mean (SD), mm Hg }\end{array}$ & $71(11)$ & $72(10)$ & $70(11)$ & $72(11)$ & 0.116 \\
\hline $\begin{array}{l}\text { Body mass index, mean } \\
\text { (SD), } \mathrm{kg} / \mathrm{m}^{2}\end{array}$ & $26.4(4.2)$ & $26.2(4.4)$ & $24.3(3.7)$ & $23.9(3.4)$ & $\hat{0.001}$ \\
\hline $\begin{array}{l}\text { Neck circumference, mean } \\
(\text { SD), cm }\end{array}$ & $39.4(3.4)$ & $39.1(3.5)$ & $38.0(3.1)$ & 37.7 (3.0) & $\dot{\delta} 001$ \\
\hline $\begin{array}{l}\text { Waist circumference, mean } \\
\text { (SD), cm }\end{array}$ & $\begin{array}{l}96.8 \\
(10.4)\end{array}$ & $95.4(11.7)$ & $91.0(9.7)$ & $89.2(9.2)$ & $\begin{array}{l}<.001 \\
0.001\end{array}$ \\
\hline \multicolumn{6}{|l|}{$\begin{array}{l}\text { Cardiovascular risk factors, } n \\
(\%)\end{array}$} \\
\hline Smoking & $73(24.7)$ & $66(30.3)$ & $82(29.5)$ & $73(33.8)$ & 0.028 \\
\hline Hyperlipidaemia & $\begin{array}{l}258 \\
(87.5)\end{array}$ & $159(72.9)$ & $239(86.0)$ & $\begin{array}{l}162 \\
(75.0)\end{array}$ & $\dot{\delta} 001$ \\
\hline Hypertension & $\begin{array}{l}255 \\
(86.4)\end{array}$ & 149 (68.3) & $219(78.8)$ & $\begin{array}{l}130 \\
(60.2)\end{array}$ & $\stackrel{<}{<.001}$ \\
\hline \multicolumn{6}{|c|}{ Abbreviation: $\mathrm{DM}$, diabetes mellitus; IQR, interquartile range; SD, standard deviation } \\
\hline \#Serum estimated glomeru & t. & (א) & $1.73 \mathrm{~m}^{2}$ & & \\
\hline
\end{tabular}




\begin{tabular}{|c|c|c|c|c|c|}
\hline Characteristics & $\begin{array}{l}\text { Sleep } \\
\text { apnea (+) } \\
\text { DM (+) } \\
(n=295)\end{array}$ & $\begin{array}{l}\text { Sleep apnea } \\
(+) \text { DM }(-) \\
(n=218)\end{array}$ & $\begin{array}{l}\text { Sleep apnea } \\
(-) \text { DM }(+) \\
(n=278)\end{array}$ & $\begin{array}{l}\text { Sleep } \\
\text { apnea }(-) \\
\text { DM }(-) \\
(n=216)\end{array}$ & $\begin{array}{l}P \\
\text { value }\end{array}$ \\
\hline Insulin dependence & $\begin{array}{l}148 \\
(50.1)\end{array}$ & - & $133(47.8)$ & - & 0.233 \\
\hline \multicolumn{6}{|l|}{$\begin{array}{l}\text { Concomitant conditions, } \mathrm{n} \\
\text { (\%) }\end{array}$} \\
\hline $\begin{array}{l}\text { Previous myocardial } \\
\text { infarction }\end{array}$ & $\begin{array}{l}145 \\
(49.2)\end{array}$ & $94(43.1)$ & $123(44.2)$ & $98(45.4)$ & 0.522 \\
\hline $\begin{array}{l}\text { Previous percutaneous } \\
\text { coronary intervention }\end{array}$ & $78(26.4)$ & $41(18.1)$ & $60(21.6)$ & $41(19.0)$ & 0.118 \\
\hline $\begin{array}{l}\text { Previous coronary artery } \\
\text { bypass surgery }\end{array}$ & $0(0.0)$ & $0(0.0)$ & $1(0.4)$ & $1(0.5)$ & 0.543 \\
\hline $\begin{array}{l}\text { Stroke/transient ischemic } \\
\text { attack }\end{array}$ & $33(11.2)$ & $28(12.8)$ & $40(14.4)$ & $17(7.9)$ & 0.148 \\
\hline Chronic kidney disease\# & $74(25.1)$ & $28(12.8)$ & $49(17.6)$ & $14(6.5)$ & $\begin{array}{l}< \\
0.001\end{array}$ \\
\hline $\begin{array}{l}\text { Chronic kidney disease on } \\
\text { dialysis }\end{array}$ & $22(7.5)$ & $6(2.8)$ & $8(2.9)$ & $0(0.0)$ & $\begin{array}{l}< \\
0.001\end{array}$ \\
\hline Pre-existing atrial fibrillation & $13(4.4)$ & $11(5.0)$ & $15(5.4)$ & $8(3.7)$ & 0.826 \\
\hline Pacemaker in-situ & $1(0.3)$ & $1(0.5)$ & $0(0.0)$ & $1(0.5)$ & 0.745 \\
\hline $\begin{array}{l}\text { Implantable cardioverter } \\
\text { defibrillator in-situ }\end{array}$ & $3(1.0)$ & $0(0.0)$ & $1(0.4)$ & $0(0.0)$ & 0.203 \\
\hline $\begin{array}{l}\text { Abbreviation: } \mathrm{DM} \text {, diabetes } \mathrm{m} \\
\text { \#Serum estimated glomerula }\end{array}$ & itus; IQR, int & quartile range; & $\begin{array}{l}\text { standard devi } \\
/ 1.73 \mathrm{~m}^{2}\end{array}$ & & \\
\hline
\end{tabular}

\section{Sleep Study Results}

The results of the sleep apnea screenings and sleep studies are listed by group in Table 2. As expected, patients in the two sleep apnea (+) groups had a higher AHI, oxygen desaturation index, and respiratory disturbance index and experienced a longer oxygen saturation duration of $<90 \%$ than did patients in the two sleep apnea (-) groups, regardless of the DM status. The four groups had similar scores on the Epworth Sleepiness Scale, a measure of daytime sleepiness, and similar results in the Berlin Questionnaire. 
Table 2

Sleep study and echocardiography results

\begin{tabular}{|c|c|c|c|c|c|}
\hline Characteristics & $\begin{array}{l}\text { Sleep } \\
\text { apnea } \\
(+) \\
\text { DM }(+) \\
(n=295)\end{array}$ & $\begin{array}{l}\text { Sleep apnea } \\
(+) \text { DM }(-) \\
(n=218)\end{array}$ & $\begin{array}{l}\text { Sleep apnea } \\
(-) \text { DM }(+) \\
(n=278)\end{array}$ & $\begin{array}{l}\text { Sleep } \\
\text { apnea } \\
(-) \\
\text { DM }(-) \\
(n=216)\end{array}$ & $\begin{array}{l}P \\
\text { value }\end{array}$ \\
\hline \multicolumn{6}{|l|}{ Sleep study } \\
\hline $\begin{array}{l}\text { AHI, events per hour, median } \\
(\mathrm{IQR})\end{array}$ & $\begin{array}{l}31.3 \\
(22.0- \\
46.1)\end{array}$ & $\begin{array}{l}27.9(19.5- \\
43.6)\end{array}$ & $\begin{array}{l}5.9(3.2- \\
10.4)\end{array}$ & $\begin{array}{l}5.75 \\
(2.8-10)\end{array}$ & $<.001$ \\
\hline $\begin{array}{l}\text { RDI, events per hour, median } \\
\text { (IQR) }\end{array}$ & $\begin{array}{l}34.6 \\
(25.2- \\
47.9)\end{array}$ & $\begin{array}{l}30.7(23.4- \\
47.0)\end{array}$ & $\begin{array}{l}9.5(6.2- \\
13.9)\end{array}$ & $\begin{array}{l}11.5 \\
(7.5- \\
15.6)\end{array}$ & $<.001$ \\
\hline $\begin{array}{l}\text { ODI, events per hour, median } \\
\text { (IQR) }\end{array}$ & $\begin{array}{l}19.9 \\
(12.1- \\
36.1)\end{array}$ & $\begin{array}{l}15.7(10.2- \\
29.8)\end{array}$ & $2.5(1.1-5.0)$ & $\begin{array}{l}2.1 \\
(0.7- \\
4.5)\end{array}$ & $<.001$ \\
\hline $\begin{array}{l}\text { Sleep duration, hour, median } \\
\text { (IQR) }\end{array}$ & $\begin{array}{l}6.5(5.4- \\
7.3)\end{array}$ & $6.3(5.6-7.2)$ & $6.3(5.4-7.0)$ & $\begin{array}{l}6.4 \\
(5.3- \\
7.3)\end{array}$ & 0.501 \\
\hline $\begin{array}{l}\text { Duration } \mathrm{SpO} 2<90 \% \text {, min, } \\
\text { median (IQR) }\end{array}$ & $\begin{array}{l}5.8(0.8- \\
24.6)\end{array}$ & $\begin{array}{l}3.7(0.3- \\
14.0)\end{array}$ & $0.0(0.0-0.5)$ & $\begin{array}{l}0.0 \\
(0.0- \\
0.6)\end{array}$ & $\langle .001$ \\
\hline $\begin{array}{l}\text { Percentage of sleep SpO } 2<90 \% \text {, } \\
\% \text {, median (IQR) }\end{array}$ & $\begin{array}{l}1.5(0.2- \\
6.3)\end{array}$ & $1.0(0.1-3.9)$ & $0.0(0.0-0.1)$ & $\begin{array}{l}0.0 \\
(0.0- \\
0.2)\end{array}$ & $<.001$ \\
\hline $\begin{array}{l}\text { Epworth Sleepiness Scale, } \\
\text { median (IQR) }{ }^{\star}\end{array}$ & $\begin{array}{l}5.0(3.0- \\
8.0)\end{array}$ & $6.0(3.0-9.0)$ & $5.0(2.0-8.0)$ & $\begin{array}{l}4.5 \\
(2.0- \\
7.0)\end{array}$ & 0.066 \\
\hline $\begin{array}{l}\text { High-risk Berlin Questionnaire, n } \\
(\%)^{\star}\end{array}$ & $\begin{array}{l}139 \\
(47.3)\end{array}$ & $100(46.1)$ & $120(43.3)$ & $\begin{array}{l}81 \\
(37.7)\end{array}$ & 0.157 \\
\hline \multicolumn{6}{|l|}{ Transthoracic echocardiography } \\
\hline $\begin{array}{l}\text { Left ventricular ejection fraction, } \\
\text { median (IQR), \% }\end{array}$ & $\begin{array}{l}48(35- \\
60)\end{array}$ & $55(42.8-60)$ & $55(45-61)$ & $\begin{array}{l}55(45- \\
60)\end{array}$ & $<.001$ \\
\hline $\begin{array}{l}\text { Left ventricular ejection fraction, } \\
\mathrm{n}(\%)\end{array}$ & & & & & $\begin{array}{l}<.001 \\
0.001\end{array}$ \\
\hline
\end{tabular}

Abbreviation: $\mathrm{AHI}$, apnea-hypopnea index; $\mathrm{BQ}$, Berlin Questionnaire; DM, diabetes mellitus; $\mathrm{ESS}$, Epworth Sleepiness Scale; ODI, oxygen desaturation index; RDI, respiratory disturbance index; SD, standard deviation; $\mathrm{SpO}_{2}$, arterial oxygen saturation.

*Four patients did not undergo the questionnaires 


\begin{tabular}{|c|c|c|c|c|c|}
\hline Characteristics & $\begin{array}{l}\text { Sleep } \\
\text { apnea } \\
(+) \\
\text { DM (+) } \\
(n=295)\end{array}$ & $\begin{array}{l}\text { Sleep apnea } \\
(+) \text { DM }(-) \\
(n=218)\end{array}$ & $\begin{array}{l}\text { Sleep apnea } \\
(-) \text { DM (+) } \\
(n=278)\end{array}$ & $\begin{array}{l}\text { Sleep } \\
\text { apnea } \\
(-) \\
\text { DM }(-) \\
(n=216)\end{array}$ & $\begin{array}{l}P \\
\text { value }\end{array}$ \\
\hline$>50 \%$ & $\begin{array}{l}112 \\
(41.3)\end{array}$ & $111(57.2)$ & $142(57.5)$ & $\begin{array}{l}122 \\
(65.9)\end{array}$ & \\
\hline $30-50 \%$ & $\begin{array}{l}105 \\
(38.7)\end{array}$ & $53(27.3)$ & 77 (31.2) & $\begin{array}{l}43 \\
(23.2)\end{array}$ & \\
\hline$<30 \%$ & 54 (19.9) & $30(15.5)$ & $28(11.3)$ & $\begin{array}{l}20 \\
(10.8)\end{array}$ & \\
\hline $\begin{array}{l}\text { Left atrium diameter, median } \\
\text { (IQR), mm }\end{array}$ & $\begin{array}{l}41(37- \\
45)\end{array}$ & $40(36-44)$ & $38(35-42)$ & $\begin{array}{l}38(35- \\
42)\end{array}$ & <. 001 \\
\hline $\begin{array}{l}\text { Left ventricular end-diastolic } \\
\text { internal diameter, } \\
\text { median (IQR), mm }\end{array}$ & $\begin{array}{l}52(47- \\
56)\end{array}$ & $52(48-57)$ & $49(45-53)$ & $\begin{array}{l}55(46- \\
55)\end{array}$ & $<.001$ \\
\hline $\begin{array}{l}\text { Left ventricular end-systolic } \\
\text { internal diameter, } \\
\text { median (IQR), mm }\end{array}$ & $\begin{array}{l}37(31- \\
45)\end{array}$ & $35(31-42)$ & $33(28-39.5)$ & $\begin{array}{l}33(29- \\
39.5)\end{array}$ & $<.001$ \\
\hline $\begin{array}{l}\text { Left ventricular mass index, } \\
\text { median (IQR), } \mathrm{g} / \mathrm{m}^{2}\end{array}$ & $\begin{array}{l}109(91- \\
132.5)\end{array}$ & $\begin{array}{l}104.5(87.8- \\
132.5)\end{array}$ & $\begin{array}{l}99(81.3- \\
120.8)\end{array}$ & $\begin{array}{l}96.5 \\
(83- \\
127)\end{array}$ & <. 001 \\
\hline $\begin{array}{l}\text { Aortic root diameter, median } \\
\text { (IQR), mm }\end{array}$ & $\begin{array}{l}33(30- \\
35)\end{array}$ & $33(31-36)$ & $32(30-35)$ & $\begin{array}{l}33(31- \\
35)\end{array}$ & 0.003 \\
\hline E/A, median (IQR) & $\begin{array}{l}0.9(0.7- \\
1.4)\end{array}$ & $0.8(0.7-1.3)$ & $0.8(0.7-1.2)$ & $\begin{array}{l}0.9 \\
(0.7- \\
1.3)\end{array}$ & 0.564 \\
\hline $\begin{array}{l}\text { Pulmonary artery systolic } \\
\text { pressure, median (IQR), mmHg }\end{array}$ & $\begin{array}{l}32 \\
(25.6- \\
43)\end{array}$ & $\begin{array}{l}28.3(23.5- \\
35.2)\end{array}$ & $28(24-34)$ & $\begin{array}{l}29(24- \\
35)\end{array}$ & 0.005 \\
\hline \multicolumn{6}{|c|}{$\begin{array}{l}\text { Abbreviation: AHI, apnea-hypopnea index; } \mathrm{BQ} \text {, Berlin Questionnaire; } \mathrm{DM} \text {, diabetes mellitus; } \mathrm{ESS} \text {, } \\
\text { Epworth Sleepiness } \mathrm{Scale}^{\mathrm{O}} \mathrm{ODI} \text {, oxygen desaturation index; } \mathrm{RDI} \text {, respiratory disturbance index; } \mathrm{SD} \text {, } \\
\text { standard deviation; } \mathrm{SpO}_{2} \text {, arterial oxygen saturation. } \\
\text { *Four patients did not undergo the questionnaires }\end{array}$} \\
\hline
\end{tabular}

\section{Echocardiography}

The echocardiography results are listed in Table 2. Patients in the sleep apnea (+) groups had a higher frequency of severe left ventricular dysfunction (left ventricular ejection fraction of $<30 \%$ ) and a greater 
left ventricular mass index, left atrial diameter, and left ventricular diameter than those in the sleep apnea $(-)$ groups, regardless of the DM status.

\section{Angiographic And Cabg Characteristics}

Details of coronary angiography findings and CABG are provided in Table 3. No significant differences were observed between the four groups with regard to the indications for angiography, number of diseased coronary vessels, and involvement of the left main and proximal left anterior descending arteries. Most patients had triple vessel disease, consistent with the current indications for CABG. More than $90 \%$ of the patients underwent conventional on-pump CABG with cardiopulmonary bypass and grafting of the left internal mammary artery to the left anterior descending artery. No significant differences were observed between the four groups in terms of the CABG characteristics, number of bypass grafts, and estimated blood loss. However, both DM (-) groups were more likely to undergo a concurrent valve operation than the DM (+) groups, regardless of the sleep apnea status. 
Table 3

Coronary angiography and CABG characteristics

\begin{tabular}{lllll}
\hline Characteristics & $\begin{array}{l}\text { Sleep } \\
\text { apnea (+) } \\
\text { DM (+) } \\
(\mathbf{n = 2 9 5 )}\end{array}$ & $\begin{array}{l}\text { Sleep } \\
\text { apnea (+) } \\
\text { DM (-) } \\
(\mathbf{n}=218)\end{array}$ & $\begin{array}{l}\text { Sleep } \\
\text { apnea (-) } \\
\text { DM (+) } \\
(\mathbf{n}=278)\end{array}$ & $\begin{array}{l}\text { Sleep apn } \\
(-) \text { DM }(-) \\
(\mathbf{n}=216)\end{array}$ \\
\hline $\begin{array}{l}\text { Clinical Presentation, } \mathbf{n}(\%) \\
\begin{array}{l}\text { ST-segment elevation } \\
\text { myocardial infarction }\end{array}\end{array}$ & $42(14.3)$ & $18(8.3)$ & $28(10.1)$ & $21(9.8)$ \\
$\begin{array}{l}\text { Non-ST-segment elevation } \\
\text { myocardial infarction }\end{array}$ & $118(40.3)$ & $87(39.9)$ & $101(36.3)$ & $84(39.1)$ \\
\hline $\begin{array}{l}\text { Unstable angina } \\
\text { Stable angina }\end{array}$ & $48(16.4)$ & $46(21.1)$ & $60(21.6)$ & $43(20.0)$ \\
\hline Other & $73(24.9)$ & $56(25.7)$ & $86(30.9)$ & $57(26.5)$ \\
\hline
\end{tabular}

\section{Number of diseased coronary \\ vessels, $n$ (\%)}

One

Two

Three

Left main artery stenosis $\geq$ $50 \%$

Proximal left anterior descending artery stenosis $\geq$ $50 \%$

\section{Operation type, n (\%)}

On-pump CABG

Off-pump CABG

Hybrid CABG

\section{Number of bypass grafts, $\mathrm{n}$}

(\%)

$$
1-2
$$

3-4

5-6

$10(3.4)$

28 (9.5)

257 (87.1)

94 (32.0)

208 (71.5)

$10(4.6)$

28 (12.8)

180 (82.6)

61 (28.2)

171 (79.2)

207 (75.0)

$5(1.8)$

34 (12.2)

78 (28.1)
0.138

\subsection{6}

$9(4.2)$

34 (15.7)

$239(86.0) \quad 173(80.1)$

79 (36.6)

0.161

$161(74.5)$ 0.272

0.234

$289(98.0) \quad 213(97.7) \quad 267(96.4) \quad 204(94.4)$

6 (2.0)

4 (1.8)

10 (3.6)

11 (5.1)

0 (0.0)

$1(0.5)$

$0(0.0)$

$1(0.5)$

Abbreviation: $\mathrm{CABG}$, coronary artery bypass grafting; $\mathrm{DM}$, diabetes mellitus; LIMA, left internal mammary artery; RIMA, right internal mammary artery; SD, standard deviation 


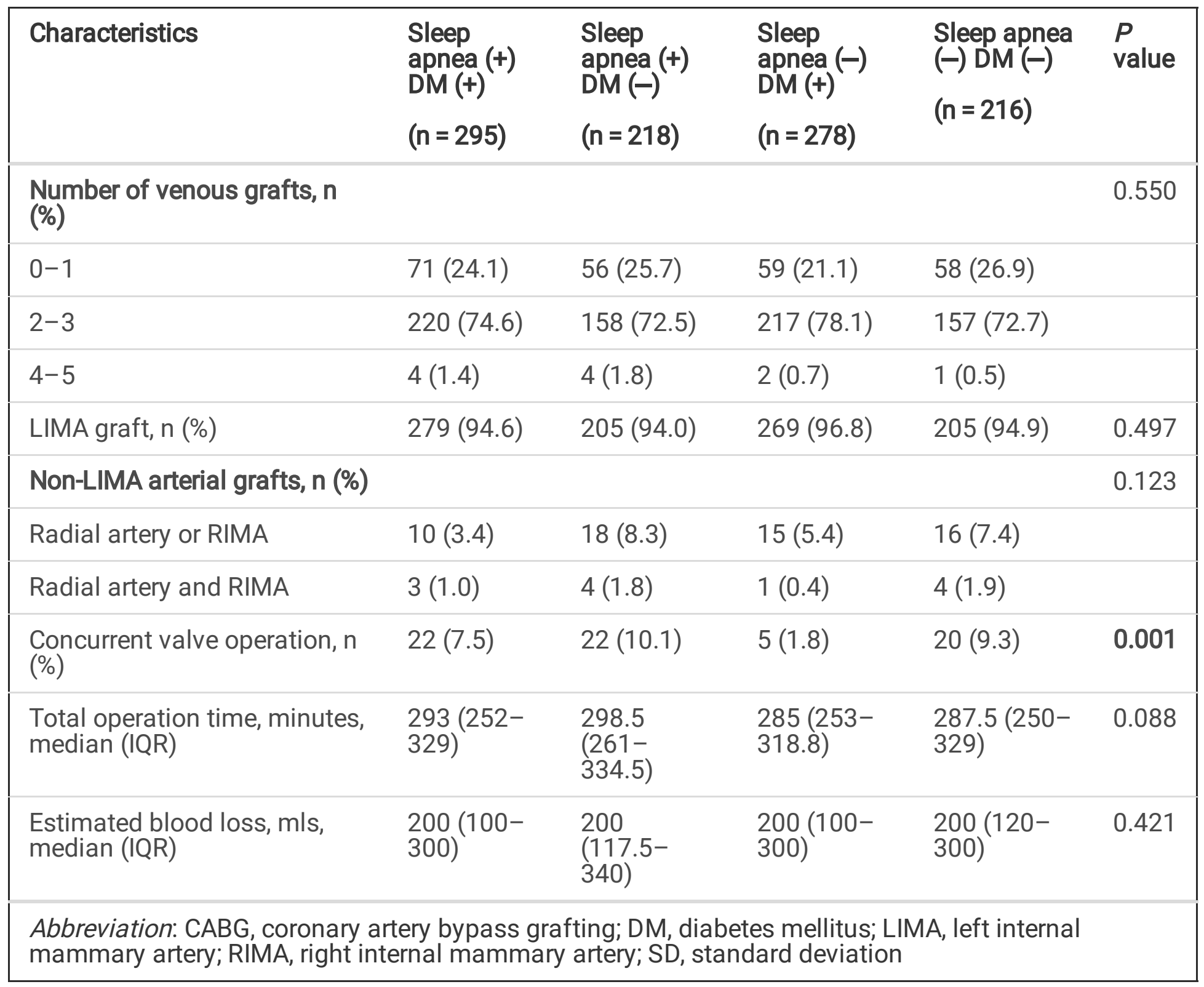

\section{Medications Upon Discharge}

Details about the medications used at the time of discharge are provided in Table 4. Most of the patients were using aspirin, $\beta$ blockers, and statins at discharge. Patients in both sleep apnea (+) groups were more likely to use frusemide, spironolactone, and warfarin than were those in both sleep apnea $(-)$ groups, regardless of the DM status. 
Table 4

Medications upon discharge

\begin{tabular}{|c|c|c|c|c|c|}
\hline Medications & $\begin{array}{l}\text { Sleep apnea (+) } \\
\text { DM (+) } \\
(n=295)\end{array}$ & $\begin{array}{l}\text { Sleep apnea (+) } \\
\text { DM }(-) \\
(n=218)\end{array}$ & $\begin{array}{l}\text { Sleep } \\
\text { apnea }(-) \\
\text { DM (+) } \\
(n=278)\end{array}$ & $\begin{array}{l}\text { Sleep apnea (-) } \\
\text { DM }(-) \\
(n=216)\end{array}$ & $\begin{array}{l}P \\
\text { value }\end{array}$ \\
\hline Aspirin & 268 (92.4) & 199 (93.0) & 245 (89.4) & 196 (91.2) & 0.482 \\
\hline$\beta$-blocker & $264(91.0)$ & $193(90.2)$ & 249 (90.9) & 192 (89.3) & 0.915 \\
\hline ACEI/ARB & $112(38.6)$ & $64(29.9)$ & $104(38.0)$ & 65 (30.2) & 0.063 \\
\hline Statin & $279(96.2)$ & 210 (98.1) & 271 (98.9) & 209 (97.2) & 0.190 \\
\hline Ezetimibe & $7(2.4)$ & $4(1.9)$ & $2(0.7)$ & $5(2.3)$ & 0.438 \\
\hline Fibrates & $7(2.4)$ & $5(2.3)$ & $7(2.6)$ & $1(0.5)$ & 0.338 \\
\hline \multicolumn{6}{|l|}{$\begin{array}{l}\text { Oral } \\
\text { anticoagulant }\end{array}$} \\
\hline Warfarin & $40(13.8)$ & $26(12.1)$ & $20(7.3)$ & $17(7.9)$ & 0.036 \\
\hline $\begin{array}{l}\text { Direct oral } \\
\text { anticoagulant }\end{array}$ & $9(3.1)$ & $8(3.7)$ & $5(1.8)$ & $3(1.4)$ & 0.342 \\
\hline Frusemide & $176(60.7)$ & $126(58.9)$ & $139(50.7)$ & $101(47.0)$ & 0.006 \\
\hline Spironolactone & $23(7.9)$ & $17(7.9)$ & $8(2.9)$ & $10(4.7)$ & 0.032 \\
\hline
\end{tabular}

\section{Follow-up And Endpoints}

Over a mean follow-up period of 2.1 years, 124 patients experienced MACCEs, including 24 cases of cardiovascular disease-related mortality, 52 cases of non-fatal myocardial infarction, and 20 cases of non-fatal stroke and unplanned revascularization. The crude incidence of MACCEs was highest in the sleep apnea (+) DM (+) group (18\%), followed by that in the sleep apnea (-) DM (+) group (13\%) and the sleep apnea (+) DM (-) group (11\%), and lowest in the sleep apnea (-) DM (-) group (5\%). Comparisons of the Kaplan-Meier cumulative incidence curves for MACCEs between the four groups are shown in Fig. 1. The Kaplan-Meier cumulative incidence curves for cardiovascular mortality, non-fatal myocardial infarction, non-fatal stroke, and hospitalization for heart failure are presented in Fig. 2.

The results of a multivariate Cox regression analysis of adverse cardiovascular events across the four groups are presented in Table 5. Using the sleep apnea (-) DM (-) group as the reference, the Cox regression analysis indicated that a sleep apnea (+) DM (+) status was associated with an increased risk 
of MACCEs (adjusted hazard ratio: $3.2,95 \%$ confidence interval: $1.7-6.2, p=0.005$ ), including increased risks of both unplanned revascularization (adjusted hazard ratio: 3.6, 95\% confidence interval: 1.0-12.5, $p=0.038$ ) and hospitalization for heart failure (adjusted hazard ratio: 12.6, 95\% confidence interval: $3.0-$ $52.3, p<0.001)$. Our analysis further revealed an association of sleep apnea with MACCEs in patients with either a DM (+) status (adjusted hazard ratio: $3.2,95 \%$ confidence interval: $1.7-6.2, p<0.001$ ) or a DM (-) status (adjusted hazard ratio: 2.1, 95\% confidence interval: $1.0-4.4, p=0.043$, interaction $p$ value: $0.473)$.

Table 5

Adjusted Hazard Ratios for adverse cardiovascular events via Cox regression

\begin{tabular}{|c|c|c|c|c|c|}
\hline Characteristics & $\begin{array}{l}\text { Sleep apnea } \\
(+) \\
\text { DM (+) } \\
(n=295)\end{array}$ & $\begin{array}{l}\text { Sleep apnea } \\
(+) \\
\text { DM }(-) \\
(n=218)\end{array}$ & $\begin{array}{l}\text { Sleep apnea } \\
(-) \\
\text { DM (+) } \\
(n=278)\end{array}$ & $\begin{array}{l}\text { Sleep } \\
\text { apnea } \\
(-) \\
\text { DM }(-) \\
(n=216)\end{array}$ & $\begin{array}{l}P \\
\text { Value }\end{array}$ \\
\hline & $\begin{array}{l}\text { Adjusted HR } \\
(95 \% \mathrm{Cl})\end{array}$ & $\begin{array}{l}\text { Adjusted HR } \\
(95 \% \mathrm{Cl})\end{array}$ & $\begin{array}{l}\text { Adjusted HR } \\
\text { ( } 95 \% \mathrm{Cl})\end{array}$ & & \\
\hline MACCE & $3.2(1.7,6.2)$ & $2.1(1.0,4.4)$ & $2.4(1.2,4.6)$ & Ref & 0.005 \\
\hline Cardiovascular mortality & $\begin{array}{l}3.2(0.9 \\
11.0)\end{array}$ & $1.9(0.5,7.4)$ & $1.9(0.5,7.2)$ & Ref & 0.250 \\
\hline Non-fatal myocardial infarction & $2.5(0.8,7.6)$ & $1.5(0.4,5.4)$ & $2.4(0.8,7.1)$ & Ref & 0.324 \\
\hline Non-fatal stroke & $1.5(0.6,3.9)$ & $1.0(0.3,2.9)$ & $1.4(0.5,3.8)$ & Ref & 0.668 \\
\hline Unplanned revascularization & $\begin{array}{l}3.6(1.0 \\
12.5)\end{array}$ & $2.2(0.5,8.7)$ & $\begin{array}{l}3.3(0.9 \\
11.6)\end{array}$ & Ref & 0.038 \\
\hline All-cause mortality & $2.6(1.0,6.8)$ & $1.6(0.5,4.7)$ & $2.0(0.7,5.6)$ & Ref & 0.230 \\
\hline $\begin{array}{l}\text { Sudden cardiac death or } \\
\text { resuscitated cardiac arrest }\end{array}$ & $\begin{array}{l}3.6(0.8 \\
16.2)\end{array}$ & $\begin{array}{l}2.7(0.5 \\
13.6)\end{array}$ & $1.9(0.4,9.4)$ & Ref & 0.289 \\
\hline Heart failure hospitalization & $\begin{array}{l}12.6(3.0 \\
52.3)\end{array}$ & $\begin{array}{l}4.4(0.9 \\
19.9)\end{array}$ & $\begin{array}{l}7.6(1.8 \\
32.7)\end{array}$ & Ref & $\begin{array}{l}<.001 \\
0.001\end{array}$ \\
\hline New-onset atrial fibrillation & $1.0(0.7,1.5)$ & $1.1(0.7,1.7)$ & $0.8(0.5,1.2)$ & Ref & 0.535 \\
\hline $\begin{array}{l}\text { Abbreviation: DM, diabetes mellit } \\
\mathrm{Cl} \text { indicates confidence interval; a } \\
\text { *Models adjusted for age, sex, bo } \\
\text { chronic kidney disease, and exce }\end{array}$ & $\begin{array}{l}\text { Is; MACCE, maj } \\
\text { hd HR, hazard }\end{array}$ & $\begin{array}{l}\text { r adverse cardi } \\
\text { tio. }\end{array}$ & and cerebrov & scular even & \\
\hline
\end{tabular}

\section{Discussion}


In this secondary analysis of 1007 patients from the SABOT study who participated in an overnight sleep study before non-emergent CABG, we observed a high prevalence of concomitant sleep apnea and DM (29.3\%). During a mean follow-up period of 2.1 years, we determined that a status of concomitant sleep apnea and DM was associated independently with a 3.2-fold increase in the risk of the four-component MACCE (cardiovascular mortality, non-fatal myocardial infarction, non-fatal stroke, and unplanned revascularization) and a 12.6-fold increase in the risk of hospitalization for heart failure. This analysis should increase awareness of the global burden of sleep apnea and DM by providing data that can facilitate the development of strategies and health policies to address this important public health problem. Our research also highlights substantial gaps in pre-CABG risk stratification, which should be very concerning because of the high prevalence and wide-ranging negative sequelae of both sleep apnea and DM.

In recent decades, the prevalence of DM has been increasing among adults worldwide. In 2017, approximately 5 million deaths among adults worldwide were attributable to DM [22]. In the same year, the total global healthcare expenditure related to DM was estimated to be USD 850 billion [22]. The prevalence of DM in Singapore is much higher than the global average (12.8\% versus $8.3 \%$ ) [23]. Recently, clinicians have tended to refer patients with DM to CABG for required revascularization. These trends may explain why the prevalence of DM in the present study (56.9\%) was higher than those reported in earlier studies conducted in Western countries (13-31\%) [3-7]. This discrepancy and the increasing recognition of sleep apnea as an important DM comorbidity and cardiovascular risk marker inspired our secondary analysis.

Although a recent report described a bidirectional association between sleep apnea and DM [24], the combined effects of these disorders on the cardiovascular outcomes of patients undergoing CABG have not been studied. No previous study on the association between DM and CABG outcomes has reported the additional prognostic effect of sleep apnea [3-8]. Recently, two relatively large-scale studies demonstrated an independent association of sleep apnea with adverse cardiovascular events in patients undergoing CABG and major non-cardiac surgery $[16,25]$. The predictive effects of sleep apnea and DM for adverse cardiovascular events were reported to be additive in patients undergoing percutaneous coronary intervention [26]. To the best of our knowledge, however, this is the first study to report a similar association in patients undergoing CABG.

Notably, a sleep apnea (+) DM (+) status seems to exert differential effects on the outcome of hospitalization for heart failure between patients undergoing percutaneous coronary intervention and those undergoing CABG. We previously reported that a sleep apnea (+) DM (+) status was independently associated with the occurrence of MACCEs, but not with hospitalization for heart failure, in patients undergoing percutaneous coronary intervention [26]. Interestingly, in our analysis of patients undergoing CABG, we observed independent associations of a sleep apnea (+) DM (+) status with both MACCEs and hospitalization for heart failure. Particularly, our secondary analysis revealed a 12.6-fold increased risk of hospitalization for heart failure after CABG. This discrepancy between our studies is intriguing but might be explained by the higher prevalence of heart failure in the sleep apnea (+) DM (+) group, which was 
indicated by the lower left ventricular ejection fraction on echocardiography and more frequent use of diuretic, $\beta$-blocker, and angiotensin converting enzyme inhibitor/angiotensin receptor blocker therapies in the sleep apnea (+) DM (+) group relative to the other three groups in this analysis. However, the relationship between a sleep apnea (+) DM (+) status and hospitalization for heart failure did not change after adjusting for covariates such as the left ventricular ejection fraction. As hospitalization after CABG remains a clinical challenge associated with reduced patient satisfaction and escalating healthcare costs, further studies are needed to evaluate the benefits of sleep apnea screening and treatment in patients with DM undergoing CABG, as highlighted by the International Diabetes Federation's Task Force on Epidemiology and Prevention [27].

This study had several limitations that should be considered. This was a secondary analysis of a completed observational study without a proper sample size calculation, which might have affected the study power for the reported endpoints. When the SABOT study was conceptualized during 2011-2012, no existing large-scale study had subjected patients to an in-laboratory polysomnography prior to CABG. As these patients faced a high level of cardiac risk, we opted for a simple wrist-worn Watch-PAT 200 sleep monitoring device to diagnose sleep apnea safely, as this device could be worn in general cardiology wards with concurrent routine nursing care. Although the Watch-PAT 200 is not considered the goldstandard diagnostic device in the sleep community, it has been subjected to validation studies [20] and has been approved by the US Food and Drug Administration for patient use. Furthermore, information related to the diagnosis of DM was obtained from the patients' electronic medical records. All patients underwent a pre-CABG evaluation (including fasting blood glucose and glycosylated hemoglobin monitoring) at the same hospital, which used the latest American Diabetic Association criteria to diagnose DM. However, the records did not include some details related to DM, including the disease duration, microvascular complications, or medication doses and adherence. These unknown or uncaptured confounding variables might have affected the results of this study. Moreover, follow-up coronary angiography was not conducted, and therefore, we were unable to evaluate the patency of the native coronary arteries or bypass grafts. Regarding our study population, our findings cannot be extrapolated to patients undergoing emergency CABG or major non-cardiac surgeries. Moreover, women were under-represented in our study population, which was predominantly Asian. It is uncertain whether our findings could be generalized to a Western population.

\section{Conclusion}

In summary, we observed a high prevalence of concomitant sleep apnea and DM among patients undergoing non-emergent CABG. Patients with concomitant sleep apnea and DM had an increased risk of developing a MACCEs (cardiovascular mortality, non-fatal myocardial infarction, non-fatal stroke, or unplanned revascularization). Moreover, a status of concomitant sleep apnea and DM was independently associated with hospitalization for heart failure. The screening and treatment of sleep apnea in patients with DM who are undergoing CABG might effectively reduce the healthcare costs and negative effects associated with this condition. 


\section{Abbreviations}

AHI: apnea-hypopnea index; CABG: coronary artery bypass grafting; DM: diabetes mellitus; MACCEs: major adverse cardiac and cerebrovascular events; PAT: peripheral arterial tone; SABOT: sleep apnea and bypass operation

\section{Declarations}

Acknowledgements: We thank Easmed Pte Ltd for their support with sleep apnea screening.

Authors' contributions: KCY, TK, and CHL designed the study. ATA, KCT, ZC, WK, HWS, and PK contributed data. KCY, WT, and CHL drafted the statistical analysis plan. WT performed the statistical analyses. ATA, $\mathrm{KCY}, \mathrm{HWS}, \mathrm{WT}$, and $\mathrm{CHL}$ wrote the manuscript. All authors contributed to and critically revised the manuscript. All authors read and approved the final manuscript.

Funding: This study was supported by a Transition Award and Clinician Scientist Award from the National Medical Research Council of Singapore (Award numbers: NMRC/TA/012/2012; NMRC/CSAINV/002/2015). The sponsors had no role in the study design, data analysis, data interpretation, writing of the report or decision to publish the manuscript.

Availability of data and materials: The data are subject to Singapore's personal data protection laws, and restrictions have been imposed by the Domain Specific Review Board to ensure the data privacy of the study participants. Therefore, data cannot be made freely available in a public repository. Please contact the corresponding author in case of further questions.

Ethics approval and consent to participate: The studies were approved by the local institutional review board (Domain Specific Review Board-C, National Healthcare Group). All participants provided written informed consent.

Consent for publication: Not applicable.

Competing interests: None declared.

DISCLOSURE

Easmed Pte. Ltd. provided support for the overnight sleep studies but had no role in the study design, data interpretation, or manuscript writing.

\section{References}

1. Farkouh ME, Domanski M, Sleeper LA, Siami FS, Dangas G, Mack M, Yang M, Cohen DJ, Rosenberg Y, Solomon SD, Desai AS, Gersh BJ, Magnuson EA, Lansky A, Boineau R, Weinberger J, Ramanathan K, Sousa JE, Rankin J, Bhargava B, Buse J, Hueb W, Smith CR, Muratov V, Bansilal S, King S 3rd, 
Bertrand M, Fuster V. FREEDOM Trial Investigators. Strategies for multivessel revascularization in patients with diabetes. N Engl J Med. 2012;367:2375-84.

2. Head SJ, Milojevic M, Taggart DP, Puskas JD. Current practice of state-of-the-art surgical coronary revascularization. Circulation. 2017;136:1331-45.

3. Leavitt BJ, Ross CS, Spence B, Surgenor SD, Olmstead EM, Clough RA, Charlesworth DC, Kramer RS, O'Connor GT, Northern New England Cardiovascular Disease Study Group. Long-term survival of patients with chronic obstructive pulmonary disease undergoing coronary artery bypass surgery. Circulation. 2006;114(1 Suppl):I430-4.

4. Alserius $\mathrm{T}$, Hammar $\mathrm{N}$, Nordqvist T, Ivert T. Risk of death or acute myocardial infarction 10 years after coronary artery bypass surgery in relation to type of diabetes. Am Heart J. 2006;152:599-605.

5. Mohammadi S, Dagenais F, Mathieu P, Kingma JG, Doyle D, Lopez S, Baillot R, Perron J, Charbonneau E, Dumont E, Metras J, Desaulniers D, Voisine P. Long-term impact of diabetes and its comorbidities in patients undergoing isolated primary coronary artery bypass graft surgery. Circulation. 2007;116(11 Suppl):1220-5.

6. Gallagher S, Kapur A, Lovell MJ, Jones DA, Kirkwood A, Hassan S, Archbold RA, Wragg A, Uppal R, Yaqoob MM. Impact of diabetes mellitus and renal insufficiency on 5-year mortality following coronary artery bypass graft surgery: a cohort study of 4869 UK patients. Eur $\mathrm{J}$ Cardiothorac Surg. 2014;45:1075-81.

7. Holzmann MJ, Rathsman B, Eliasson B, Kuhl J, Svensson AM, Nyström T, Sartipy U. Long-term prognosis in patients with type 1 and 2 diabetes mellitus after coronary artery bypass grafting. $\mathrm{J}$ Am Coll Cardiol. 2015;65:1644-52.

8. Kogan A, Ram E, Levin S, Fisman EZ, Tenenbaum A, Raanani E, Sternik L. Impact of type 2 diabetes mellitus on short- and long-term mortality after coronary artery bypass surgery. Cardiovasc Diabetol. 2018;17:151.

9. Pamidi S, Tasali E. Obstructive sleep apnea and type 2 diabetes: is there a link? Front Neurol. 2012;3:126.

10. Reutrakul S, Mokhlesi B. Obstructive sleep apnea and diabetes: A state of the art review. Chest. 2017;152:1070-86.

11. Javaheri S, Barbe F, Campos-Rodriguez F, Dempsey JA, Khayat R, Javaheri S, Malhotra A, MartinezGarcia MA, Mehra R, Pack Al, Polotsky VY, Redline S, Somers VK. Sleep apnea: Types, mechanisms, and clinical cardiovascular consequences. J Am Coll Cardiol. 2017;69:841-58.

12. Zhao LP, Kofidis T, Lim TW, Chan SP, Ong TH, Tan HC, Lee CH. Sleep apnea is associated with newonset atrial fibrillation after coronary artery bypass grafting. J Crit Care. 2015;30:1418.e1-5.

13. Zhao LP, Kofidis T, Chan SP, Ong TH, Yeo TC, Tan HC, Lee CH. Sleep apnoea and unscheduled readmission in patients undergoing coronary artery bypass surgery. Atherosclerosis. 2015;242:128-34.

14. Rupprecht S, Schultze T, Nachtmann A, Rastan AJ, Doenst T, Schwab M, Witte OW, Rohe S, Zwacka I, Hoyer $\mathrm{H}$. Impact of sleep disordered breathing on short-term post-operative outcome after elective 
coronary artery bypass graft surgery: a prospective observational study. Eur Respir J. 2017; 49. pii: 1601486.

15. Uchôa CHG, Danzi-Soares NJ, Nunes FS, de Souza AAL, Nerbass FB, Pedrosa RP, César LAM, LorenziFilho G, Drager LF. Impact of OSA on cardiovascular events after coronary artery bypass surgery. Chest. 2015;147:1352-60.

16. Koo CY, Aung AT, Chen Z, Kristanto W, Sim HW, Tam WW, Gochuico CF, Tan K, Kang CS, Sorokin V, Ong P, Kojodjojo P, Richards AM, Tan HC, Kofidis T, Lee CH. Sleep apnoea and cardiovascular outcomes after coronary artery bypass grafting. Heart. 2020 (in press).

17. American Diabetes Association. Diagnosis and classification of diabetes mellitus. Diabetes Care. 2010;33(Suppl 1):62-9.

18. Netzer NC, Stoohs RA, Netzer CM, Clark K, Strohl KP. Using the Berlin Questionnaire to identify patients at risk for the sleep apnea syndrome. Ann Intern Med. 1999;131:485-91.

19. Johns MW. A new method for measuring daytime sleepiness: the Epworth sleepiness scale. Sleep. 1991;14:540-5.

20. Yalamanchali S, Farajian V, Hamilton C, Pott TR, Samuelson CG, Friedman M. Diagnosis of obstructive sleep apnea by peripheral arterial tonometry: meta-analysis. JAMA Otolaryngol Head Neck Surg. 2013; 139: 1343-1350.

21. Spitzer E, McFadden E, Vranckx P, Garcia-Garcia HM, Seltzer JH, Held C, de Vries T, Menon V, Brown KJ, Soliman OII, Onuma Y, Lopes RD, Stone GW, Cutlip DE, Serruys PW. Critical appraisal of contemporary clinical endpoint definitions in coronary intervention trials: A guidance document. JACC Cardiovasc Interv. 2019;12:805-19.

22. Cho NH, Shaw JE, Karuranga S, Huang Y, da Rocha Fernandes JD, Ohlrogge AW, Malanda B. IDF Diabetes Atlas: global estimates of diabetes prevalence for 2017 and projections for 2045. Diabetes Res Clin Pract. 2018;138:271-81.

23. Wong LY, Toh MP, Tham LW. Projection of prediabetes and diabetes population size in Singapore using a dynamic Markov model. J Diabetes. 2017;9:65-75.

24. Huang T, Lin BM, Stampfer MJ, Tworoger SS, Hu FB, Redline S. A population-based study of the bidirectional association between obstructive sleep apnea and type 2 diabetes in three prospective U.S. cohorts. Diabetes Care. 2018;41:2111-9.

25. Chan MTV, Wang CY, Seet E, Tam S, Lai HY, Chew EFF, Wu WKK, Cheng BCP, Lam CKM, Short TG, Hui DSC, Chung F. Postoperative Vascular Complications in Unrecognized Obstructive Sleep Apnea (POSA) Study Investigators. Association of unrecognized obstructive sleep apnea with postoperative cardiovascular events in patients undergoing major noncardiac surgery. JAMA. 2019;321:1788-98.

26. Koo CY, Drager LF, Sethi R, Ho HH, Hein T, Jim MH, Tai BC, Zhang JJ, Lee CH. Obstructive sleep apnea and diabetes mellitus independently add to cardiovascular risk after coronary revascularization. Diabetes Care. 2018;41:e12-4.

27. Shaw JE, Punjabi NM, Wilding JP, Alberti KG, Zimmet PZ. Sleep-disordered breathing and type 2 diabetes: a report from the International Diabetes Federation Taskforce on Epidemiology and 
Prevention. Diabetes Res Clin Pract. 2008;81:2-12.

\section{Figures}

1

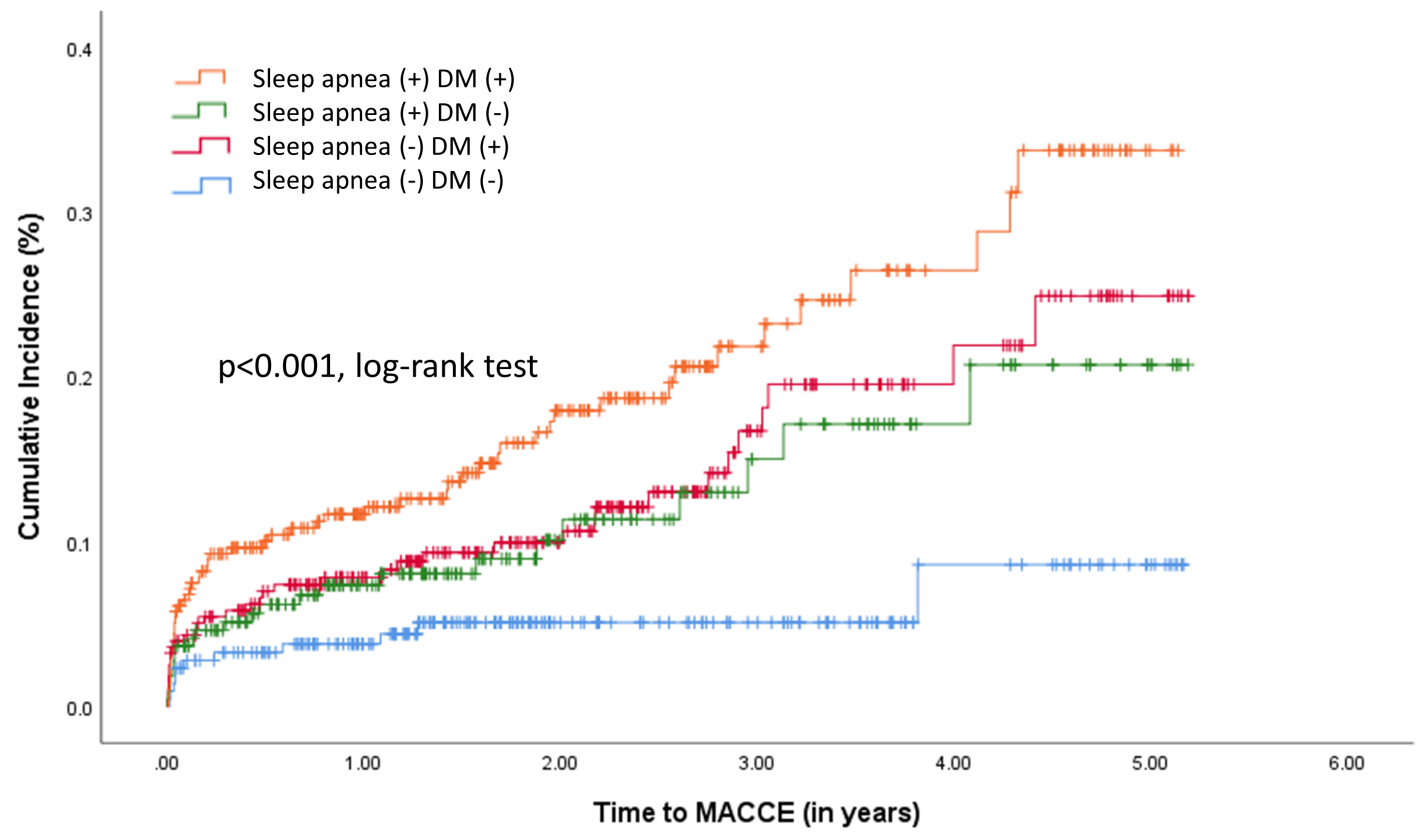

\begin{tabular}{|l|}
\hline Sleep apnea (+) DM (+) \\
\hline Sleep apnea (+) DM (-) \\
\hline Sleep apnea (-) DM (+) \\
\hline Sleep apnea (-) DM (-) \\
\hline
\end{tabular}

\begin{tabular}{|l|l|l|l|l|l|}
\hline 295 & 192 & 120 & 58 & 30 & 2 \\
\hline 218 & 137 & 71 & 39 & 22 & 5 \\
\hline 278 & 196 & 130 & 60 & 32 & 7 \\
\hline 216 & 159 & 81 & 56 & 24 & 9 \\
\hline
\end{tabular}

\section{Figure 1}

Cumulative incidence of major adverse cardiac and cerebrovascular events (MACCEs), defined as a fourcomponent composite of cardiovascular mortality, nonfatal myocardial infarction, nonfatal stroke, and unplanned revascularization. 
$2 A$

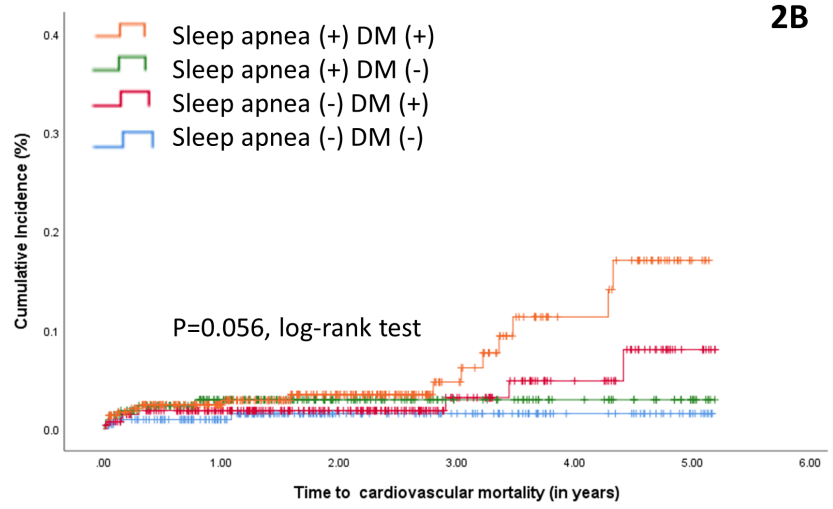

2C

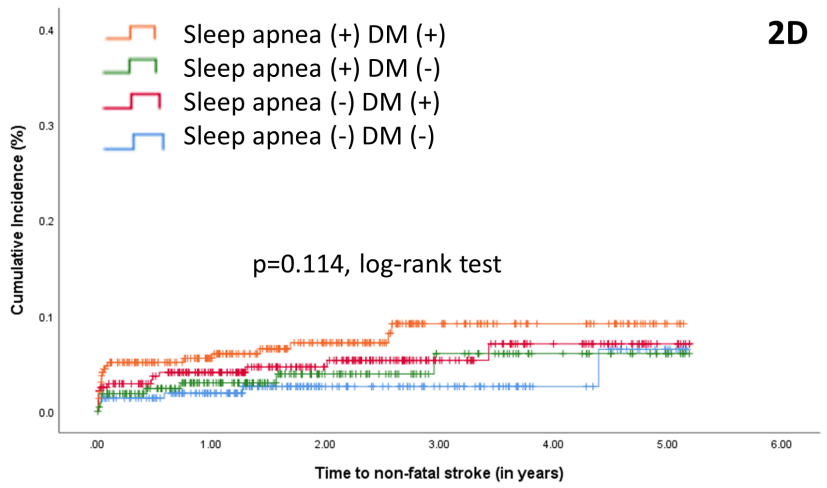

2B
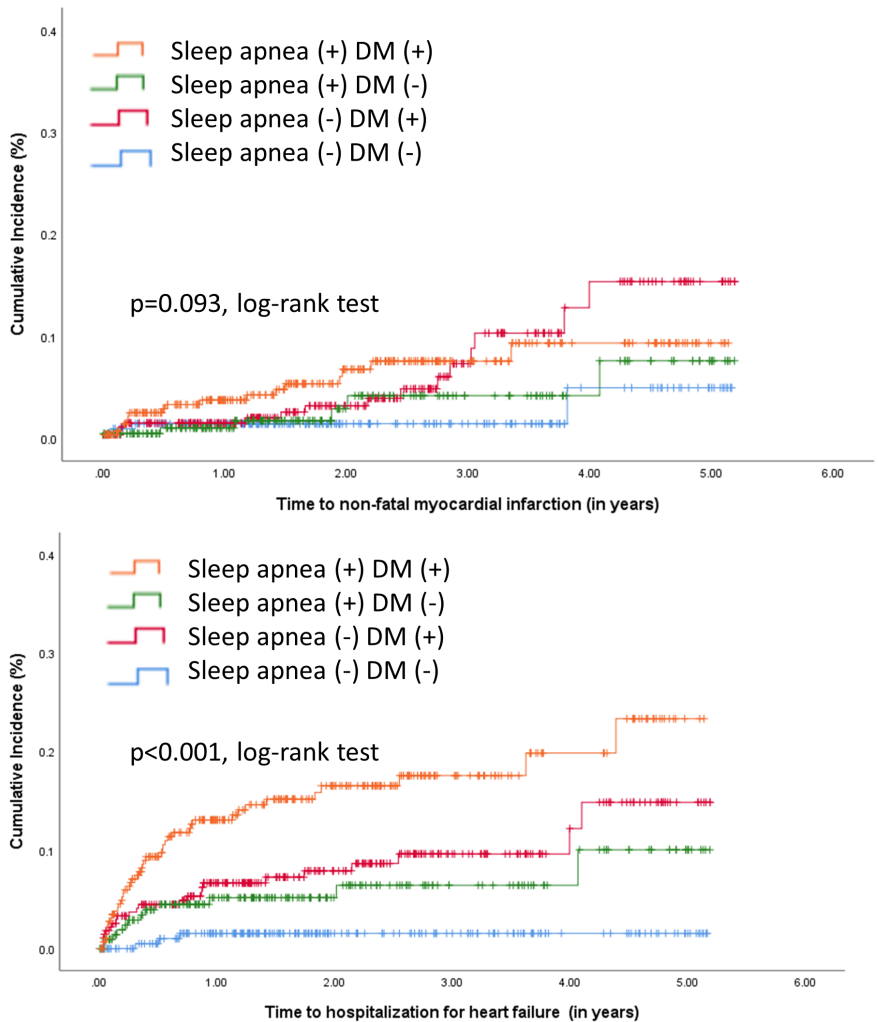

\section{Figure 2}

Kaplan-Meier analyses of the cumulative incidences of cardiovascular mortality (A), non-fatal myocardial infarction (B), non-fatal stroke (C), and hospitalization for heart failure (D). 